\title{
The Christensen measurable solutions of a generalization of the Gołąb-Schinzel functional equation
}

\author{
by JANUSz BRZDȨK (Rzeszów)
}

\begin{abstract}
Let $K$ denote the set of all reals or complex numbers. Let $X$ be a topological linear separable $F$-space over $K$. The following generalization of the result of C. G. Popa [16] is proved.

THEOREM. Let $n$ be a positive integer. If a Christensen measurable function $f: X \rightarrow$ $K$ satisfies the functional equation

$$
f\left(x+f(x)^{n} y\right)=f(x) f(y)
$$

then it is continuous or the set $\{x \in X: f(x) \neq 0\}$ is a Christensen zero set.
\end{abstract}

1. Introduction. The functional equation

$$
f(x+f(x) y)=f(x) f(y)
$$

is well known and has been studied by many authors (see e.g. [1], [2], [4], [5], [11]-[13], [15], [16], [19]). It is called the Gołąb-Schinzel functional equation. C. G. Popa [16] has proved that every Lebesgue measurable solution $f: \mathbb{R} \rightarrow$ $\mathbb{R}$ of (1) is either continuous or equal to zero almost everywhere. We are going to show that the same is true for each Christensen measurable solution of the functional equation

$$
f\left(x+f(x)^{n} y\right)=f(x) f(y)
$$

mapping a real (complex) linear topological separable $F$-space into the set of all reals (complex numbers), where $n$ is a positive integer.

Equation (2) is a natural generalization of (1). It is also a particular case $(k=0, t=1)$ of the functional equation

$$
f\left(f(y)^{k} x+f(x)^{n} y\right)=t f(x) f(y)
$$

considered in various cases e.g. in [3], [4], [7], [18]. It is also worth mentioning that there is a strict connection between the solutions of equation (2) in the

1991 Mathematics Subject Classification: Primary 39B52.

Key words and phrases: Gołąb-Schinzel functional equation, Christensen measurability, $F$-space. 
class of functions $f: \mathbb{R} \rightarrow \mathbb{R}$ and a class of subgroups of the Lie group $L_{n+1}^{1}$ (cf. [5], [6]).

Throughout this paper $\mathbb{N}, \mathbb{Z}, \mathbb{R}$, and $\mathbb{C}$ will denote the sets of all positive integers, all integers, reals, and complex numbers, respectively. $X$ stands for a linear space over a field $K \in\{\mathbb{R}, \mathbb{C}\}$, unless explicitly stated otherwise. $m$ and $m_{\mathrm{i}}$ are Lebesgue and inner Lebesgue measures in $K$, respectively.

2. Preliminary lemmas. Let us start with the following

Lemma 1. A function $f: X \rightarrow K, f \neq 0$ (i.e. $f^{-1}(\{0\}) \neq X$ ), is a solution of equation (2) iff there exist an additive subgroup $A$ of $X, a$ multiplicative subgroup $W$ of $K$, and a function $w: W \rightarrow X$ such that

(3) $a^{n} A=A \quad$ for $a \in W$;

(4) $\quad w(a b)-a^{n} w(b)-w(a) \in A \quad$ for $a, b \in W$;

(5) $w(a) \in A$ iff $a=1$;

(6) $f(x)=\left\{\begin{array}{ll}a & \text { if } x \in w(a)+A \text { and } a \in W, \\ 0 & \text { otherwise, }\end{array}\right.$ for $x \in X$.

Furthermore, $W=f(X) \backslash\{0\}$ and $A=f^{-1}(\{1\})$.

The proof does not differ essentially from the proof of Theorem 1 of [13] (cf. also [19] and [12], pp. 275-277). Therefore we omit it.

The subsequent corollary follows from Lemma 1.

Corollary 1. If a function $f: X \rightarrow K, f \neq 0$, satisfies equation (2), $A=f^{-1}(\{1\})$, and $W=f(X) \backslash\{0\}$, then:

(i) $A$ is an additive group;

(ii) $W$ is a multiplicative group;

(iii) $A \backslash\{0\}$ is the set of periods of $f$;

(iv) if $x, y \in X$ and $f(x)=f(y) \neq 0$, then $x-y \in A$;

(v) $a^{n} A=A$ for $a \in W$.

Lemma 2. Let $f: K \rightarrow K$ be a microperiodic function (i.e. the set of periods of $f$ is dense in $K$ ) satisfying equation (2). Suppose that there exists $a \in K$ such that $|f(a)| \notin\{0,1\}$. Then $m_{\mathrm{i}}\left(f^{-1}\left(K_{j}\right)\right)=0$ for $j \in \mathbb{N}$, where $K_{j}=\{a \in K: 1 / j \leq|a| \leq j\}$.

Proof. For an indirect proof suppose that there is $k \in \mathbb{N}$ with $m_{\mathrm{i}}\left(f^{-1}\left(K_{k}\right)\right)>0$. Then, in view of Corollary $1(\mathrm{ii})$, there exists $b \in K$ with $|f(b)|>(k+1)^{2}$. Put $D=b+f(b)^{n} f^{-1}\left(K_{k}\right)$. It is easily seen that $m_{\mathrm{i}}(D)>0$ and, by (2), $|f(a)|>k$ for $a \in D$. Thus $D \cap f^{-1}\left(K_{k}\right)=\emptyset$. On the other hand, according to a theorem of H. Steinhaus (see e.g. [14], Theorem 3.7.1), $\operatorname{int}\left(D-f^{-1}\left(K_{k}\right)\right) \neq \emptyset$. Consequently, there exists $c \in D-f^{-1}\left(K_{k}\right)$ such 
that $c \neq 0$ and $f(a+c)=f(a)$ for $a \in K$, which means that $f^{-1}\left(K_{k}\right) \cap D=$ $\left(f^{-1}\left(K_{k}\right)+c\right) \cap D \neq \emptyset$, a contradiction.

Given $b \in \mathbb{C} \backslash\{0\}$ and $j \in \mathbb{N}$ let us put

$$
C_{j}(b)=\left\{a \in \mathbb{C} \backslash\{0\}:(j-1) \frac{2}{3} \pi \leq \operatorname{Arg} b^{-1} a<j \frac{2}{3} \pi\right\},
$$

where $\operatorname{Arg} c \in[0,2 \pi)$ denotes the argument of $c \in \mathbb{C} \backslash\{0\}$. It is easy to see that $\mathbb{C} \backslash\{0\}=\bigcup\left\{C_{j}(b): j=1,2,3\right\}$.

Lemma 3. Let $f: \mathbb{C} \rightarrow \mathbb{C}$ be a microperiodic solution of (2) such that the set $f(\mathbb{C})$ is infinite and $|a|=1$ for $a \in f(\mathbb{C}) \backslash\{0\}$. Then $m_{\mathrm{i}}\left(f^{-1}\left(C_{j}(b)\right)\right)=0$ for every $j=1,2,3, b \in \mathbb{C} \backslash\{0\}$.

Proof. For an indirect proof suppose that there exist $b \in \mathbb{C} \backslash\{0\}$ and $k \in\{1,2,3\}$ with $m_{\mathrm{i}}\left(f^{-1}\left(C_{k}(b)\right)\right)>0$. Since $f(\mathbb{C})$ is infinite, in view of Corollary 1 (ii), $f(\mathbb{C}) \backslash\{0\}$ is dense in the set $J=\{a \in \mathbb{C}:|a|=1\}$. Thus there is $d \in \mathbb{C}$ such that $f(d) \neq 0$ and $\left(f(d) C_{k}(b)\right) \cap C_{k}(b)=\emptyset$. Define $D=d+f(d)^{n} f^{-1}\left(C_{k}(b)\right)$. Then, in virtue of $(2), f(D)=f(d) C_{k}(b)$. Hence $D \cap f^{-1}\left(C_{k}(b)\right)=\emptyset$. On the other hand, $m_{\mathrm{i}}(D)>0$, which, according to the theorem of Steinhaus, means that $\operatorname{int}\left(D-f^{-1}\left(C_{k}(b)\right)\right) \neq \emptyset$. Consequently, there exists a period $c \in D-f^{-1}\left(C_{k}(b)\right)$ of $f$, from which we derive that $f^{-1}\left(C_{k}(b)\right) \cap D=\left(f^{-1}\left(C_{k}(b)\right)+c\right) \cap D \neq \emptyset$, a contradiction.

Lemma 4. If a function $f: K \rightarrow K, f \neq 1$, satisfies equation (2), then $m_{\mathrm{i}}\left(f^{-1}(\{a\})\right)=0$ for each $a \in f(K) \backslash\{0\}$.

Proof. For an indirect proof suppose that there is $a \in f(K) \backslash\{0\}$ with $m_{\mathrm{i}}\left(f^{-1}(\{a\})\right)>0$. Fix $b \in f^{-1}(\{a\})$ and put $D=f^{-1}(\{a\})-b$. Then, on account of Corollary 1(iv), $D \subset A:=f^{-1}(\{1\})$. Thus $m_{\mathrm{i}}(A)>0$. Consequently, by the theorem of Steinhaus and Corollary 1(i), $A=K$, a contradiction.

Lemma 5. Let $f: X \rightarrow K$ be a function satisfying equation (2), $W=$ $f(X) \backslash\{0\}$, and $A=f^{-1}(\{1\})$. Suppose that there is $a_{0} \in W$ such that $a_{0}^{n} \neq 1$ and $\left(a_{0}^{n}-1\right)^{-1} A \subset A$. Then

$$
a^{n} \neq 1 \quad \text { for each } a \in W \backslash\{1\}
$$

and there exists $x_{0} \in X \backslash \bigcup\left\{\left(a^{n}-1\right)^{-1} A: a \in W \backslash\{1\}\right\}$ such that

$$
f(x)=\left\{\begin{array}{ll}
a & \text { if } x \in\left(a^{n}-1\right) x_{0}+A \text { and } a \in W, \\
0 & \text { otherwise, }
\end{array} \text { for } x \in X .\right.
$$

Proof. In view of Lemma 1 there is a function $w: W \rightarrow X$ such that (4)-(6) hold. Let $x_{0}=\left(a_{0}^{n}-1\right)^{-1} w\left(a_{0}\right)$. Since, by (4),

$$
w(a b)-a^{n} w(b)-w(a), w(b a)-b^{n} w(a)-w(b) \in A \quad \text { for } a, b \in W,
$$

Corollary 1(i) implies that $a^{n} w(b)+w(a)-b^{n} w(a)-w(b) \in A$ for $a, b \in W$. Thus, for each $b \in W,-\left(b^{n}-1\right) x_{0}+w(b) \in A$. Consequently, according to 
(5), (6), and Corollary 1(i), conditions (8) and (9) hold and $\left(a^{n}-1\right) x_{0} \notin A$ for $a \in W \backslash\{1\}$, which completes the proof.

Lemma 6. Let $Y$ be a linear space over a subfield $F$ of the field $K$. Let $f: Y \rightarrow K \backslash\{0\}$ be a solution of equation (2) such that $f(x)^{n} \in F$ for each $x \in Y$. Then $f=1$.

Proof. Suppose that there is $x \in Y$ with $f(x)^{n} \neq 1$ and put $z=$ $\left(1-f(x)^{n}\right)^{-1} x$. Then $x+f(x)^{n} z=z$ and, in view of $(2)$,

$$
f(x) f(z)=f\left(x+f(x)^{n} z\right)=f(z) \neq 0,
$$

from which we derive $f(x)=1$, a contradiction.

Hence $f(x)^{n}=1$ for each $x \in Y$. Thus $f(x+y)=f(x) f(y)$ for $x, y \in Y$ and consequently, for each $x \in Y$,

$$
f(x)=f\left(n \frac{1}{n} x\right)=f\left(\frac{1}{n} x\right)^{n}=1 .
$$

This completes the proof.

Lemma 7. If a function $f: X \rightarrow K, f \neq 0$, satisfies equation (2), then $f\left(f(x)^{-n}(z-x)\right)=f(z) f(x)^{-1}$ for $x, z \in X$ with $f(x) \neq 0$.

Proof. Fix $x \in X$ with $f(x) \neq 0$. Setting $z=f(x)^{n} y+x$ in (2), we get $f(z)=f(x) f\left(f(x)^{-n}(z-x)\right)$ for $z \in X$, which yields the assertion.

Lemma 8. Let $B$ be an additive subgroup of a real linear space $Y$ and let $V$ be an infinite multiplicative subgroup of $\mathbb{R}$ such that

$$
a x \in B \quad \text { for } x \in B, a \in V .
$$

Then the set $B_{x}=\{a \in \mathbb{R}: a x \in B\}$ is dense in $\mathbb{R}$ for each $x \in B$.

Proof. Note that, for each $c \in \mathbb{R}, c>0$, there is $b \in V$ with $|b|<c$. Since, for each $x \in B, B_{x}$ is an additive group and, by (10), $V \subset B_{x}$, we obtain the statement.

Lemma 9. Let $B$ be an additive subgroup of a complex linear space and let $V$ be an infinite multiplicative subgroup of $\mathbb{C}$ such that $V \not \subset \mathbb{R}$ and (10) holds. Then the set $B_{x}=\{a \in \mathbb{C}: a x \in B\}$ is dense in $\mathbb{C}$ for each $x \in B$.

Proof. Let $x \in B$ and $J=\{a \in \mathbb{C}:|a|=1\}$. Note that $V \subset B_{x}$. If $V \subset J$, then $V$ is dense in $J$. Thus $B_{x}$ is dense in $\mathbb{C}$, because it is an additive group. On the contrary, if there is $a \in V \backslash(R \cup J)$, then, for each $c \in \mathbb{R}$, $c>0$, there exists $k \in \mathbb{Z}$ with $\left|a^{k}\right|<c$ and $\left|a^{k+1}\right|<c$. Since $a^{k}$ and $a^{k+1}$ are linearly independent over $\mathbb{R}$, the additive group generated by $V$ is dense in $\mathbb{C}$, which completes the proof.

Lemma 10 (cf. [16], Théorème 1 ). If $D_{1}, D_{2} \subset K$ and $m_{\mathrm{i}}\left(D_{j}\right)>0$, $j=1,2$, then $\operatorname{int}\left(D_{1} \cdot D_{2}\right) \neq \emptyset$. 
Pro of. First consider the case where $K=\mathbb{R}$. There exist closed sets $F_{i} \subset$ $D_{i}$ such that $m\left(F_{i}\right)>0$ for $i=1,2$. Put $F_{i}^{k}=F_{i} \cap([-k,-1 / k] \cup[1 / k, k])$ for $k \in \mathbb{N}, i=1,2$. It is easily seen that there are $p, q \in \mathbb{N}$ with $m\left(F_{1}^{p}\right)>0$ and $m\left(F_{2}^{q}\right)>0$. Let $F_{1}^{+}=F_{1}^{p} \cap(0, \infty), F_{1}^{-}=F_{1}^{p} \cap(-\infty, 0), F_{2}^{+}=F_{2}^{q} \cap(0, \infty)$, and $F_{2}^{-}=F_{2}^{q} \cap(-\infty, 0)$. Define

$$
F_{i}^{0}=\left\{\begin{array}{ll}
F_{i}^{+} & \text {if } m\left(F_{i}^{+}\right)>0, \\
F_{i}^{-} & \text {otherwise },
\end{array} \quad \text { for } i=1,2 .\right.
$$

Observe that $m\left(F_{i}^{0}\right)>0$ for $i=1,2$. Thus (see e.g. [17], Theorem 8.26), $m\left(\ln F_{i}^{0}\right)>0$ for $i=1,2$. Hence, in virtue of the theorem of Steinhaus, $\operatorname{int}\left(\ln \left(F_{1}^{0} \cdot F_{2}^{0}\right)\right)=\operatorname{int}\left(\ln F_{1}^{0}+\ln F_{2}^{0}\right) \neq \emptyset$, which means that $\operatorname{int}\left(D_{1} \cdot D_{2}\right) \neq \emptyset$.

Now assume that $K=\mathbb{C}$. Let $F_{i} \subset D_{i}$ be a closed set such that $m\left(F_{i}\right)>$ 0 for $i=1,2$. Put $C_{k}=\{a \in \mathbb{C}: 1 / k \leq|a| \leq k\}$ and $F_{i}^{k}=F_{i} \cap C_{k}$ for $k \in \mathbb{N}, i=1,2$. It is easily seen that there are $p, q \in \mathbb{N}$ with $m\left(F_{1}^{p}\right)>0$ and $m\left(F_{2}^{q}\right)>0$. Define functions $h_{1}: \mathbb{R} \times(\mathbb{R} \backslash\{0\}) \rightarrow \mathbb{C}, h_{2}:(0,2 \pi) \times(0, \infty) \rightarrow \mathbb{C}$, and $h_{3}: \mathbb{R} \times(0, \infty) \rightarrow \mathbb{R}^{2}$ by the formulas: $h_{1}(a, b)=a+i b, h_{2}(a, b)=$ $b(\cos a+i \sin a), h_{3}(a, b)=(a, \ln b)$. Let $F_{1}^{0}=h_{1}^{-1}\left(F_{1}^{p}\right)$ and $F_{2}^{0}=h_{1}^{-1}\left(F_{2}^{q}\right)$. Then $F_{i}^{0}$ is a Borel set and $m\left(F_{i}^{0}\right)>0$ for $i=1,2(m$ denotes also the Lebesgue measure in $\left.\mathbb{R}^{2}\right)$. Note that $h=h_{3} \circ h_{2}^{-1} \circ h_{1}$ is a diffeomorphism onto the set $h(\mathbb{R} \times(\mathbb{R} \backslash\{0\}))$. Thus $h\left(F_{i}^{0}\right)$ is a Borel set and $m\left(h\left(F_{i}^{0}\right)\right)>0$ for $i=1,2$ (see e.g. [17], Theorem 8.26(c)). Hence, by the theorem of Steinhaus, $\operatorname{int}\left(h\left(F_{1}^{0}\right)+h\left(F_{2}^{0}\right)\right) \neq \emptyset$. Since $h\left(F_{1}^{0}\right)+h\left(F_{2}^{0}\right)=h_{3} \circ h_{2}^{-1}\left(h_{1}\left(F_{1}^{0}\right) h_{1}\left(F_{2}^{0}\right)\right)$, we have $\operatorname{int}\left(h_{1}\left(F_{1}^{0}\right) \cdot h_{1}\left(F_{2}^{0}\right)\right) \neq \emptyset$, which implies the assertion.

3. Christensen measurability. Throughout this part we assume that $X$ is a separable $F$-space as a topological linear space over $K$. We shall use the notation and terminology from [8]-[10] concerning Christensen measurability. Now, we only recall necessary definitions and facts.

Let $M$ be the $\sigma$-algebra of all universally measurable subsets of $X$; i.e. $M$ is the intersection of all completions of the Borel $\sigma$-algebra of $X$ with respect to probability Borel measures. In the following a measure is a countable additive Borel measure extended to $M$.

Definition 1. A set $B \in M$ is a Haar zero set iff there exists a probability measure $u$ on $X$ such that $u(B+x)=0$ for each $x \in X$.

Definition 2. A set $P \subset X$ is a Christensen zero set iff it is a subset of a Haar zero set.

Definition 3. A set $D \subset X$ is Christensen measurable iff $D=B \cup P$, where $B \in M$ and $P$ is a Christensen zero set. 
Let us define

$$
\begin{aligned}
\mathcal{C}_{0} & =\{B \subset X: B \text { is Christensen zero set }\} \\
\mathcal{C} & =\{B \subset X: B \text { is Christensen measurable }\} .
\end{aligned}
$$

LEMma 11 (see [9], Theorem 1). Every countable union of Christensen zero sets is a Christensen zero set.

Lemma 12 (see [9], Theorem 2). If $B \in \mathcal{C} \backslash \mathcal{C}_{0}$, then $0 \in \operatorname{int}(B-B)$.

Definition 4. A function $f: X \rightarrow K$ is said to be Christensen measurable iff $f^{-1}(U) \in \mathcal{C}$ for each open set $U \subset K$.

Lemma 13 (see [10], Theorem 1). Let $f: X \rightarrow K$ be a Christensen measurable linear functional. Then $f$ is continuous.

Put $L_{k}=\{a \in K: k-1 \leq|a|<k\}$ and $a_{k}=m\left(L_{k}\right)$ for $k \in \mathbb{N}$. Given a Borel set $D \subset X$ and $x \in X$ denote $u_{x}(D)=m_{p}\left(k_{x}^{-1}(D)\right)$, where $k_{x}: K \rightarrow X, k_{x}(a)=a x$, and, for each Borel set $B \subset K, m_{p}(B)=$ $\sum_{k=1}^{\infty} 2^{-k} a_{k}^{-1} m\left(B \cap L_{k}\right)$. Since $k_{x}$ is continuous, $u_{x}$ is a well defined Borel measure and $u_{x}(X)=1$ for each $x \in X \backslash\{0\}$.

Lemma 14. Let $D \in \mathcal{C} \backslash \mathcal{C}_{0}$ and $x \in X \backslash\{0\}$. Then there exist a Borel set $D_{x} \subset D$ and $y_{x} \in X$ such that

$$
m\left(k_{x}^{-1}\left(y_{x}+D_{x}\right)\right)>0 .
$$

Pr o of. There exist $B \in M$ and $P \in \mathcal{C}_{0}$ with $D=B \cup P$. In view of Lemma $11, B \notin \mathcal{C}_{0}$. Thus there is $y \in X$ such that $\bar{u}(B+y)>0$, where $\bar{u}$ denotes the extension of $u_{x}$ to $M$. Put $u_{0}(T)=\bar{u}(T+y)$ for each $T \in M$. Then $u_{0}$ is a probability measure. Hence there are a Borel set $B_{x} \subset B$ and a set $B_{0} \subset B$ such that $u_{0}\left(B_{0}\right)=0$ and $B=B_{x} \cup B_{0}$. Furthermore $u_{x}\left(B_{x}+y\right)=\bar{u}\left(B_{x}+y\right)=u_{0}\left(B_{x}\right)=u_{0}\left(B_{x} \cup B_{0}\right)=u_{0}(B)=\bar{u}(B+y)>0$. Consequently, $m_{p}\left(k_{x}^{-1}\left(B_{x}+y\right)\right)>0$, which implies (11). This ends the proof.

LEMmA 15. Let $L \subset K \backslash\{0\}$ and $x \in X \backslash\{0\}$. Let $f: X \rightarrow K$ be a function satisfying equation (2). Suppose that $f^{-1}(L) \in \mathcal{C} \backslash \mathcal{C}_{0}$. Then there exists $z \in X$ such that $f(z) \neq 0$ and $m_{\mathrm{i}}\left(f_{x}^{-1}\left(f(z)^{-1} L\right)\right)>0$, where $f_{x}: K \rightarrow K$, $f_{x}(a)=f(a x)$.

P r o of. It follows from Lemma 14 that there are a Borel set $D_{x} \subset D:=$ $f^{-1}(L)$ and $y_{x} \in X$ such that (11) holds. Put $B=\left(K x-y_{x}\right) \cap D_{x}$. Then, according to the definition of $k_{x}$ and (11), $B \neq \emptyset$. Fix $z \in B$. It is easily seen that $f(z) \neq 0$ and there exists $b \in K$ with $z=b x-y_{x}$. Thus

$$
B-z=\left(\left(K x-y_{x}\right) \cap D_{x}\right)-b x+y_{x}=\left(K x \cap\left(D_{x}+y_{x}\right)\right)-b x,
$$

which means that $k_{x}^{-1}(B-z)=k_{x}^{-1}\left(D_{x}+y_{x}\right)-b$. Hence, in view of (11),

$$
m\left(f(z)^{-n}\left(k_{x}^{-1}(B-z)\right)\right)>0 .
$$


Note that, by Lemma 7 ,

$$
\begin{aligned}
f_{x}\left(f(z)^{-n}\left(k_{x}^{-1}(B-z)\right)\right) & =f\left(f(z)^{-n}\left(k_{x}^{-1}(B-z)\right) x\right) \subset f\left(f(z)^{-n}(B-z)\right) \\
& =f(z)^{-1} f(B) \subset f(z)^{-1} L .
\end{aligned}
$$

Consequently, $f(z)^{-n}\left(k_{x}^{-1}(B-z)\right) \subset f_{x}^{-1}\left(f(z)^{-1} L\right)$, from which we derive by $(12)$, that $m_{\mathrm{i}}\left(f_{x}^{-1}\left(f(z)^{-1} L\right)\right)>0$. This completes the proof.

Lemma 16. Let $f: X \rightarrow K$ be a Christensen measurable function satisfying equation (2) such that the set $W=f(X) \backslash\{0\}$ is infinite. Suppose that the set $S_{f}=\{x \in X: f(x) \neq 0\}$ is not a Christensen zero set. Then the set $A=f^{-1}(\{1\})$ is a proper linear subspace of $X$ over the field

$$
F= \begin{cases}\mathbb{R} & \text { if } f(x)^{n} \in \mathbb{R} \text { for each } x \in X \\ \mathbb{C} \quad \text { otherwise. }\end{cases}
$$

Pr o of. Since $A \neq X$, it suffices to show that $A$ is a linear subspace of $X$ over $F$.

For an indirect proof suppose that $A \neq A_{0}$, where $A_{0}$ denotes the linear subspace of $X$ (over $F$ ) spanned by $A$. Let $f_{0}=\left.f\right|_{A_{0}}$. It is easy to check that $f_{0}$ is a solution of $(2)$ and $f_{0} \neq 1$. Thus, in view of Lemma $6, f_{0}^{-1}(\{0\}) \neq \emptyset$, from which we derive that there are $a_{0} \in F \backslash\{0\}$ and $y \in A \backslash\{0\}$ such that $f\left(a_{0} y\right)=0$. Note that the functions $f_{1}: X \rightarrow F, f_{1}(x)=f(x)^{n}$, and $f_{y}$ : $F \rightarrow F, f_{y}(a)=f_{1}(a y)$, also satisfy (2) for $n=1$. Since $f_{y}\left(a_{0}\right)=f\left(a_{0} y\right)^{n}=$ 0 , we have $f_{y} \neq 1$. Furthermore, $W_{n} \subset F,\{a \in F: a y \in A\} \subset f_{y}^{-1}(\{1\})$, and, by Corollary $1(\mathrm{v}), a A=A$ for $a \in W_{n}$, where $W_{n}=\left\{a^{n}: a \in W\right\}$. Hence, by Lemma 8, Lemma 9 , and Corollary 1(i)-(iii), $f_{y}$ is microperiodic.

First consider the case where there is $b \in F$ with $\left|f_{y}(b)\right| \notin\{0,1\}$. Let $F_{j}=\{a \in F: 1 / j \leq|a| \leq j\}$ for $j \in \mathbb{N}$. Since $S_{f}=\bigcup\left\{f_{1}^{-1}\left(F_{j}\right): j \in \mathbb{N}\right\}$, according to Lemma 11 there exists $p \in \mathbb{N}$ such that $f_{1}^{-1}\left(F_{p}\right) \notin \mathcal{C}_{0}$. Thus, by Lemma 15 (with $n=1$ ), $m_{\mathrm{i}}\left(f_{y}^{-1}\left(f_{1}(z)^{-1} F_{p}\right)\right)>0$ for some $z \in S_{f}$. Note that there is $k \in \mathbb{N}$ with $f_{1}(z)^{-1} F_{p} \subset F_{k}$. Hence $m_{\mathrm{i}}\left(f_{y}^{-1}\left(F_{k}\right)\right)>0$, which contradicts Lemma 2.

Now, assume that the set $W_{y}:=f_{y}(F) \backslash\{0\}$ is finite. Then $W_{y}$ is a multiplicative cyclic subgroup of $F$ (cf. Corollary 1(ii)) and $|a|=1$ for each $a \in W_{y}$. There exists $c \in F$ such that $W_{y}=\left\{c^{k}: k \in \mathbb{N}\right\}$. Put $k_{0}=\min \left\{k \in \mathbb{N}: c^{k}=1\right\}$ and define

$$
T_{j}= \begin{cases}c^{j}(0, \infty) & \text { if } F=\mathbb{R} \\ \left\{a \in \mathbb{C} \backslash\{0\}: 2 \pi k_{0}^{-1}(j-1) \leq \operatorname{Arg} a<2 \pi k_{0}^{-1} j\right\} & \text { if } F=\mathbb{C}\end{cases}
$$

for $j \in \mathbb{N}, j \leq k_{0}$. Observe that $S_{f}=\bigcup\left\{f_{1}^{-1}\left(T_{j}\right): j \in \mathbb{N}, j \leq k_{0}\right\}$. Thus there is a positive integer $k \leq k_{0}$ such that $f_{1}^{-1}\left(T_{k}\right) \notin \mathcal{C}_{0}$. It results from Lemma 15 that there exists $z \in S_{f}$ with $m_{\mathrm{i}}\left(f_{y}^{-1}\left(f_{1}(z)^{-1} T_{k}\right)\right)>0$. Moreover, there is exactly one positive integer $p \leq k_{0}$ such that $c^{p} \in f_{1}(z)^{-1} T_{k}$. Consequently, $m_{\mathrm{i}}\left(f_{y}^{-1}\left(\left\{c^{p}\right\}\right)\right)>0$, contrary to Lemma 4 . 
It remains to study the case where $F=\mathbb{C}, W_{y}$ is infinite, and $|a|=1$ for each $a \in W_{y}$. Since $S_{f}=\bigcup\left\{f_{1}^{-1}\left(C_{j}(1)\right): j=1,2,3\right\}$, where $C_{j}(b)$, for $b \in \mathbb{C} \backslash\{0\}$, is given by $(7)$, we have $f_{1}^{-1}\left(C_{k}(1)\right) \notin \mathcal{C}_{0}$ for some $k \in\{1,2,3\}$. Thus, on account of Lemma 15 , there is $z \in S_{f}$ with $m_{\mathrm{i}}\left(f_{y}^{-1}\left(f_{1}(z)^{-1} C_{k}(1)\right)\right)$ $>0$. Clearly, $f_{1}(z)^{-1} C_{k}(1)=C_{k}\left(f_{1}(z)^{-1}\right)$. Hence $m_{\mathrm{i}}\left(f_{y}^{-1}\left(C_{k}\left(f_{1}(z)^{-1}\right)\right)\right)>0$, contrary to Lemma 3 . This completes the proof.

4. The main result. Now, we have all tools to prove the announced theorem.

TheOREM. Suppose that $X$ is a linear topological separable F-space over $K$. Let $f: X \rightarrow K$ be a Christensen measurable solution of equation (2). Then either $f$ is continuous or the set $S_{f}=\{x \in X: f(x) \neq 0\}$ is a Christensen zero set.

Furthermore, if $f$ is continuous and satisfies (2), then

$$
f(X) \subset \mathbb{R} \quad \text { or } \quad n=1
$$

and the following two statements hold:

(i) if $f(X) \subset \mathbb{R}$, then there exists a continuous $\mathbb{R}$-linear functional $g$ : $X \rightarrow \mathbb{R}$ such that, for $n$ odd, either

$$
f(x)=\sqrt[n]{g(x)+1} \quad \text { for } x \in X
$$

or

$$
f(x)=\sqrt[n]{\sup (g(x)+1,0)} \quad \text { for } x \in X
$$

and for $n$ even, $f$ is of the form (16);

(ii) if $f(X) \not \subset \mathbb{R}$ and $n=1$, then there exists a continuous $\mathbb{C}$-linear functional $g: X \rightarrow \mathbb{C}, g \neq 0$, such that $f(x)=g(x)+1, x \in X$.

Proof. Note that if $f \neq 0$ is continuous, then int $S_{f} \neq \emptyset$, which means that $S_{f} \notin \mathcal{C}_{0}$. Therefore suppose that $S_{f} \in \mathcal{C} \backslash \mathcal{C}_{0}$. Put $W=f(X) \backslash\{0\}$ and $A=f^{-1}(\{1\})$.

First, consider the case where $W$ is finite. Then, in view of Lemma 1, there is a function $w: W \rightarrow X$ with $S_{f}=\bigcup\{w(a)+A: a \in W\}$. Thus, by Lemma 11, $A \notin \mathcal{C}_{0}$. Hence Lemma 12 and Corollary 1(i) imply that $\operatorname{int} A \neq \emptyset$, from which we derive $A=X$. Consequently, (15) or (16) holds with $g=0$.

Now, assume that $W$ is infinite. Since, in the case where $K=\mathbb{C}, X$ is also a real topological linear $F$-space (with the same topology), without loss of generality we may assume that

$$
\text { if } K=\mathbb{C} \text {, then } f(X) \not \subset \mathbb{R} \text {. }
$$

It results from Lemma 16 that $A$ is a proper linear subspace of $X$ over the field $F$ given by (13). Thus, by Lemma 5 , condition (8) is valid and there 
exists $x_{0} \in X \backslash A$ such that $f$ is of the form (9). Hence

$$
S_{f}=A+\left(W_{n}-1\right) x_{0},
$$

where $W_{n}=\left\{a^{n}: a \in W\right\}$. Furthermore, in view of Lemma 12, $0 \in \operatorname{int}\left(S_{f}-\right.$ $\left.S_{f}\right)$, whence

$$
A+F x_{0}=X .
$$

On account of (19) and Lemma 14 there exist a Borel set $B \subset S_{f}$ and $a \in F$, $x \in A$ with $m\left(k_{0}^{-1}\left(a x_{0}+x+B\right)\right)>0$, where $k_{0}: F \rightarrow X, k_{0}(a)=a x_{0}$. On the other hand, from (18), we obtain $a x_{0}+x+S_{f}=A+\left(W_{n}-1+a\right) x_{0}$. Thus $k_{0}^{-1}\left(a x_{0}+x+S_{f}\right)=W_{n}-1+a$. Since $k_{0}^{-1}\left(a x_{0}+x+B\right)=a+k_{0}^{-1}(x+B)$, we have $k_{0}^{-1}(x+B) \subset W_{n}-1$ and $m\left(k_{0}^{-1}(x+B)\right)=m\left(k_{0}^{-1}\left(a x_{0}+x+B\right)\right)>0$, from which we derive that $m_{\mathrm{i}}\left(W_{n}\right)=m_{\mathrm{i}}\left(W_{n}-1\right)>0$ (in $F$ ). Hence and from Lemma 10 and Corollary 1 (ii) we get int $W_{n} \neq \emptyset$ (in $F$ ), whence

$$
\left.(0, \infty) \subset W_{n} \quad \text { and } \quad 1 \in \operatorname{int} W_{n} \text { (in } F\right) .
$$

We shall prove that (8), (17), and (20) imply $F=K$.

For an indirect proof suppose that $K=\mathbb{C}$ and $F=\mathbb{R}$. Then there is $a \in W \backslash \mathbb{R}$ with $a^{n} \in \mathbb{R}$. Observe that, by (20) and Corollary 1(ii), $a \cdot|a|^{-1} \in W \backslash \mathbb{R}$, whence, by (8), $-1=\left(a \cdot|a|^{-1}\right)^{n} \in W$ and $(-1)^{n} \neq 1$. This means that $n$ is odd. Consequently, $a^{n+1} \cdot|a|^{-n-1}=-a \cdot|a|^{-1} \notin \mathbb{R}$ and $\left(a^{n+1} \cdot|a|^{-n-1}\right)^{n}=(-1)^{n+1}=1$, which contradicts (8).

In this way we have proved that $F=K$. Thus, by (19), $A$ is a hyperplane of $X$ (i.e. codim $A=1$ ) and, according to Corollary 1 and (20),

$$
\begin{aligned}
& \text { for } K=\mathbb{C}, \quad W=\mathbb{C} \backslash\{0\}, \\
& \text { for } K=\mathbb{R}, \quad W=(0, \infty) \text { or } W=\mathbb{R} \backslash\{0\},
\end{aligned}
$$

whence (8) yields condition (14).

Define a linear functional $g: X \rightarrow K$ by

$$
g\left(a x_{0}+y\right)=a \quad \text { for } a \in K, y \in A .
$$

It is easy to check that, on account of (9) and (18),

$$
g(x)=f(x)^{n}-1 \quad \text { for } x \in S_{f},
$$

which, in view of (8), (18), and (22), means that, in the case where $f(X) \subset \mathbb{R}$, both conclusions of (i) are valid. In the case where $n=1$ and $f(X) \not \subset \mathbb{R}$, (21), (18), and (24) imply that $f(x)=g(x)+1, x \in X$. Therefore, on account of Lemma 13, it remains to show that $g$ is Christensen measurable.

If $n=1$ and $f(X) \not \subset \mathbb{R}$, this is obvious, because $f$ is Christensen measurable. On the other hand, if $f(X) \subset \mathbb{R}$, then $g(x)=f(x)^{n}-1$ for $x \in g^{-1}((-1, \infty))$. Furthermore, for each set $U \subset \mathbb{R}, g^{-1}(U)=g^{-1}\left(U^{+}\right) \cup$ $\left(-g^{-1}\left(-U^{-}\right)\right) \cup g^{-1}\left(U_{0}\right)$, where $U^{+}=U \cap(0, \infty), U^{-}=U \cap(-\infty, 0)$ and 
$U_{0}=U \cap\{0\}$. This implies that $g$ is Christensen measurable, which ends the proof.

Remark. It is easy to check that each function $f: X \rightarrow K$ satisfying (14) and conditions (i), (ii) of the Theorem is a solution of equation (2).

Finally, since in the case where $X$ is locally compact, $\mathcal{C}_{0}$ coincides with the set of all the Haar measure zero subsets of $X$ (see [9], p. 256), from the Theorem we get the following

Corollary 2. Let $k \in \mathbb{N}$ and let $f: K^{k} \rightarrow K$ be a Lebesgue measurable solution of equation (2). Then either $f$ is continuous or the set $S_{f}$ is of Lebesgue measure zero.

Acknowledgements. I wish to thank Professor Karol Baron for calling my attention to the problem.

\section{References}

[1] J. Aczél and S. Gołąb, Remarks on one-parameter subsemigroups of the affine group and their homo- and isomorphisms, Aequationes Math. 4 (1970), 1-10.

[2] K. Baron, On the continuous solutions of the Gotab-Schinzel equation, ibid. 38 (1989), 155-162.

[3] W. Benz, The cardinality of the set of discontinuous solutions of a class of functional equations, ibid. 32 (1987), 58-62.

[4] N. Brillouët et J. Dhombres, Equations fonctionnelles et recherche de sous groupes, ibid. 31 (1986), 253-293.

[5] J. Brzdęk, Subgroups of the group $Z_{n}$ and a generalization of the Gotab-Schinzel functional equation, ibid. 43 (1992), 59-71.

[6] -, A generalization of the Gotab-Schinzel functional equation, ibid. 39 (1990), 268269.

[7] -, On the solutions of the functional equation $f\left(x f(y)^{l}+y f(x)^{k}\right)=t f(x) f(y)$, Publ. Math. Debrecen 38 (1991), 175-183.

[8] J. P. R. Christensen, Topology and Borel Structure, North-Holland Math. Stud. 10, North-Holland, 1974

[9] - On sets of Haar measure zero in abelian Polish groups, Israel J. Math. 13 (1972), 255-260.

[10] P. Fischer and Z. Słodkowski, Christensen zero sets and measurable convex functions, Proc. Amer. Math. Soc. 79 (1980), 449-453.

[11] S. Gołąb et A. Schinzel, Sur l'équation fonctionnelle $f(x+y f(x))=f(x) f(y)$, Publ. Math. Debrecen 6 (1959), 113-125.

[12] D. Ilse, I. Lechmann und W. Schulz, Gruppoide und Funktionalgleichungen, Deutscher Verlag Wiss., Berlin, 1984.

[13] P. Javor, On the general solution of the functional equation $f(x+y f(x))=$ $f(x) f(y)$, Aequationes Math. 1 (1968), 235-238.

[14] M. Kuczma, An Introduction to the Theory of Functional Equations and Inequalities, PWN and Uniw. Ślasski, Warszawa-Kraków-Katowice, 1985.

[15] P. Plaumann und S. Strambach, Zweidimensionale Quasialgebren mit Nullteilern, Aequationes Math. 15 (1977), 249-264. 
[16] C. G. Popa, Sur l'équation fonctionnelle $f(x+y f(x))=f(x) f(y)$, Ann. Polon. Math. 17 (1965), 193-198.

[17] W. Rudin, Real and Complex Analysis, McGraw-Hill, 1974.

[18] M. Sablik and P. Urban, On the solutions of the equation $f\left(x f(y)^{k}+y f(x)^{l}\right)=$ $f(x) f(y)$, Demonstratio Math. 18 (1985), 863-867.

[19] S. Wołodźko, Solution générale de l'équation fonctionnelle $f(x+y f(x))=$ $f(x) f(y)$, Aequationes Math. 2 (1968), 12-29.

Institute of Mathematics

Pedagogical University of Rzeszów

Rejtana 16A

35-310 Rzeszów, Poland

Reçu par la Rédaction le 17.12.1990

Révisé le 23.10.1995 